\title{
Actividades de debate en el proceso de aprendizaje. Experiencias e implicancias desde procesos de formación de estudiantes de economía
}

Salazar Espinoza, César Antonio; Acuña, Andrés A.

Actividades de debate en el proceso de aprendizaje. Experiencias e implicancias desde procesos de formación de estudiantes de economía

Revista Educación, vol. 42, núm. 2, 2018

Universidad de Costa Rica, Costa Rica

Disponible en: http://www.redalyc.org/articulo.oa?id=44055139012

DOI: https://doi.org/10.15517/revedu.v42i2.24956

Esta obra está bajo una Licencia Creative Commons Atribución-NoComercial-SinDerivar 3.0 Internacional. 
Actividades de debate en el proceso de aprendizaje. Experiencias e implicancias desde procesos de formación de estudiantes de economía

\author{
Debate Activities in the Learning Process. Experiences and Implications from the Formation Process of Economics \\ Students
}

César Antonio Salazar Espinoza

Universidad del Bio-Bio, Chile

csalazar@ubiobio.cl

Andrés A. Acuña

Universidad del Bío-Bio, Chile

aacunad@ubiobio.cl

\author{
DOI: https://doi.org/10.15517/revedu.v42i2.24956 \\ Redalyc: http://www.redalyc.org/articulo.oa?id=44055139012
}

Recepción: 15 Junio 2016

Aprobación: 20 Marzo 2018

\title{
RESUMEN:
}

Los cambios en la dinámica que ha tomado la economía nacional y su entorno empresarial exigen una modificación en los modelos educativos y en los métodos de enseñanza desde un proceso de aprendizaje continuo basado en el estudiantado, que permita el desarrollo de competencias genéricas necesarias para el buen desempeño laboral. Este estudio tiene como objetivo diseñar, implementar y sistematizar experiencias de actividades de debate en programas de economía que se encuentran en renovación curricular con un modelo educativo centrado en el estudiantado. Las actividades se llevaron a cabo con estudiantes de economía de la Facultad de Ciencias Empresariales de la Universidad del Bío-Bío, Chile. En el diseño de las actividades, se desarrollan versiones alternativas de debates, donde se consideran variaciones en función de algunas características de los grupos. Asimismo, se señala y discute un conjunto de criterios de evaluación. Del mismo modo, se desarrolla un protocolo donde se estructuran las etapas, los detalles logísticos de la actividad y el uso del equipamiento. Los resultados sugieren que la motivación estudiantil es clave para el éxito de la actividad y propone la definición de los equipos en forma intencionada. Esta experiencia prueba que es posible realizar debates en cursos numerosos, si se definen protocolos alternativos y se entregan los incentivos con base en premios y sanciones en la dirección correcta. Se concluye que, para que los resultados sean los esperados, se requiere que este tipo de actividades se desarrollen de manera sistemática durante todo el proceso de formación. Asimismo, es necesario comprometer la participación activa del personal académico y precisar explícitamente el "debate” como una actividad formal de evaluación en los programas.

Palabras clave: Debates, metologías de enseñanza, experiencias, economía.

\section{ABstraCT:}

The change in the dynamics of the national economy and its business environment require modifications in the educational models and methodologies compatible with an ongoing learning process based on the student towards the development of necessary generic competences for a good job performance. This study aims at designing, implementing and systematizing debate experiences in course syllabuses for economics being updated following a student-centered educational model. . The activities were carried out at the Faculty of Economics and Business at the University of Bio-Bio, Chile.Alternative versions of debates based on group characteristics and a specific a set of evaluation criteria were proposed. In addition, a protocol containing the phases of the activity, logistics and equipment use was developed. The results suggest that student motivation is a key factor for a successful debate when the formation of groups is intentional. This experience proves that it is possible to carry out debates in numerous courses defining alternative protocols and giving incentives based on rewards and penalties in the correct direction. It can also be concluded that

\section{Notas DE AUTOR}

[2] PhD en Economía y académico de la Facultad de Ciencias Empresariales de la Universidad del Bío-Bío, sede Chillán, Chile. En la actualidad se desempeña como académico del Departamento de Gestión Empresarial, unidad de la cual forma parte desde el año 2018. Sus áreas de investigación son economía del desarrollo y economía de los recursos naturales.

[3] Magíster en Economía y académico de la Facultad de Ciencias Empresariales de la Universidad del Bío-Bío, sede Concepción, Chile. En la actualidad se desempeña como director del Departamento de Economía y Finanzas, unidad de la cual forma parte desde el año 2005. Sus áreas de investigación son economía financiera y macroeconomía aplicada. 
activities must be conducted systematically throughout the training process. It is also necessary to foster the active participation of academics and explicitly specify the "debate"; as a formal activity as part of the evaluation criteria in the proposed -course syllabi. KEYWORDS: debates, learning process methodologies, experiences, economics.

\section{INTRODUCCIÓN}

El mundo de hoy, globalizado, requiere de grupos profesionales creativos, proactivos y flexibles, con capacidad de liderazgo y adaptación al cambio como actitud permanente al trabajo. El entorno laboral exige que las personas se comprometan y se responsabilicen individual y colectivamente en el trabajo y en los valores y normas disciplinarias. Estos cambios que ha tomado la economía nacional y su entorno empresarial exigen una modificación de los modelos educativos y de los métodos de enseñanza en un proceso de aprendizaje continuo que se base en el estudiantado, que permita el desarrollo de ciertas competencias genéricas necesarias para el buen desempeño laboral. En efecto, la comunicación verbal efectiva es una competencia genérica de gran relevancia para quienes se insertan en el mercado laboral, mientras que el grado de desarrollo del pensamiento crítico en estudiantes de pre y postgrado será crucial en todo rol que asuman durante su futuro ejercicio profesional (Darby, 2007; Estaville, 1988). Por consiguiente, una institución de educación superior, fiel a su espíritu o misión, debe "brindar a sus estudiantes los espacios y oportunidades tanto para instruirse sobre nuevos tópicos como para agudizar su capacidad crítica” (Vo y Morris, 2006, p. 316).

En este contexto, el nuevo modelo educativo de la Universidad del Bío-Bío asume este desafío y propone una modificación en la forma de realizar la docencia, cuyo enfoque fundamental busca centrar el aprendizaje en el alumnado. De esta manera se hace necesario alinear el currículo y las técnicas de aprendizajes de las carreras impartidas por la universidad a su modelo educativo (Universidad del Bío-Bío, 2008). En particular, los planes de estudios de las carreras ofrecidas en la Facultad de Ciencias Empresariales presentan una rigidez que no se condice con los cambios en el entorno ni con el modelo educativo de la universidad. Por ejemplo, el modelo curricular de la carrera de Ingeniería Comercial data desde 1991, y contiene elementos de un modelo tradicional centrado en la enseñanza del profesorado, principalmente sobre la base de la transferencia de contenidos (Facultad de Ciencias Empresariales, 2008). Sin embargo, durante el año 2008 se ha llevado a cabo un proceso de actualización y reformulación del plan de estudios, con base en las exigencias trazadas en el nuevo modelo educativo de la universidad y que están en consonancia con las mayores exigencias de un mundo globalizado y cada vez más dinámico.

De esta manera, la motivación del estudio recae particularmente en estos cambios en los paradigmas de la práctica docente, y que se vinculan con las necesidades empresariales asociadas al desarrollo de competencias laborales genéricas en el marco de un entorno cambiante y dinámico. La Universidad del Bío-Bío incorpora estos elementos en su nuevo modelo educativo, sin embargo, aún se encuentra en una fase de restructuración que involucra no solamente el diseño curricular, sino la aplicación de este nuevo modelo educativo en el aula. Es en este contexto donde las actividades de debate cobran especial importancia como un instrumento para el desarrollo y evaluación de competencias genéricas en el aula.

Este estudio tiene por objeto diseñar, implementar y sistematizar experiencias de actividades de debate, con el propósito de determinar los elementos claves para una implementación exitosa del "debate" como un nuevo instrumento de evaluación para la praxis docente. Las actividades se llevaron a cabo en cursos de la línea economía, incorporadas en los planes curriculares de las carreras Contador Público y Auditor, Ingeniería Comercial e Ingeniería Civil Informática, las cuales forman parte de la oferta formativa de la Facultad de Ciencias Empresariales de la Universidad del Bío-Bío, Chile. Por medio de este tipo de actividades se espera reforzar la capacidad de expresión oral del alumnado, motivando su reflexión crítica con base en argumentos técnicos, la coordinación, el trabajo colaborativo y la cohesión grupal, en un escenario que responde a situaciones de presión, inesperadas y complejas; todo esto desde un marco de discrepancia y respeto. 
Complementariamente, se espera que este tipo de instancias favorezcan las relaciones socioafectivas entre estudiantes y la relación profesorado-alumnado. En el diseño de la actividad, se proponen versiones alternativas de debates que consideran variaciones en función de algunas características de los grupos. Asimismo, se señalan y discuten un conjunto de criterios de evaluación. Del mismo modo, se desarrolla un protocolo donde se estructuran las etapas, los detalles logísticos de la actividad y el uso del equipamiento. El siguiente artículo se estructura como sigue. Primero, se presenta un marco teórico general sobre conceptos relacionados con la didáctica, instrumentos de evaluación y, en particular, para el caso del debate. Posteriormente, se plantea el marco metodológico del estudio. Seguidamente, se presentan los resultados. Finalmente, se discuten las conclusiones.

\section{MARCO TEÓRICO}

Antes de comenzar con la discusión de los debates como técnica de enseñanza-aprendizaje, es menester desarrollar los conceptos de praxis docente, didáctica y evaluación que forman parte de la presente investigación.

De acuerdo con Zufiarre y Gabari (2001), la praxis docente comprende un conjunto de herramientas y técnicas empleadas por el personal académico, que configuran la didáctica, y que facilitan el proceso de enseñanza-aprendizaje, aceptando la incidencia que sobre este tienen los factores afectivos, cognitivos y sociales del alumnado. Los autores señalan que la didáctica, por su parte, se debe dimensionar como una disciplina que integra las ciencias de la educación. Etimológicamente, el término didáctica proviene del griego didaskein que significa enseñar, instruir, explicar. Es así como se concibe la didáctica como la ciencia que se aboca al estudio de la enseñanza orientada al aprendizaje (Zufiaurre y Gabari, 2001).

Desde una perspectiva histórica, se debe distinguir entre la didáctica tradicional y la didáctica moderna. La didáctica tradicional tiene sus orígenes en el racionalismo. Epistemológicamente, el racionalismo es aquella visión que apela a la razón como fuente de conocimiento, es un método o teoría en el cual el criterio para conocer la verdad no es sensitivo, sino intelectual y deductivo (Azócar, 2006).

De acuerdo con Zufiaurre y Gabari (2001), la didáctica moderna, por su parte, acepta la existencia de factores afectivos, cognitivos y sociales que inciden sobre el proceso enseñanza-aprendizaje. Dado esto, los autores distinguen los siguientes componentes:

- Componente artístico: se refiere a la habilidad del personal docente para transmitir conocimientos, la cual es espontánea, intuitiva y personal y que le permite reaccionar ante la imprevisibilidad y complejidad de la praxis didáctica.

- Componente técnico: se refiere al conjunto de procedimientos puestos en práctica por el personal docente para obtener un resultado, el aprendizaje.

- Componente tecnológico: se refiere a la aplicación de procedimientos basados en el conocimiento científico, es decir, que estos sean compatibles con el método científico.

En la evolución de la didáctica como ciencia, Chevallard (1991) plantea el abandono de la práctica tradicional, cuyos fundamentos se encuentran en el racionalismo académico, y plantea la adopción de la transposición didáctica en la praxis docente, concepto que surge de la didáctica de las matemáticas. La transposición didáctica consiste en lograr que un conocimiento duro (saber sabio) sea, en primer lugar, internalizado (saber enseñado) por estudiantes y, luego, que estos grupos o individuos sean capaces de generar nuevos conocimientos a partir de lo aprendido y de su experiencia sensorial.

$\mathrm{Al}$ respecto, Cardelli (2004) señala que el desarrollo del saber científico está asociado a la constitución de determinadas instituciones, en las cuales se dan las relaciones de los sujetos (estudiantes) con el saber. En este sentido, el proceso de aprendizaje surge de las prácticas de estudio organizadas por el profesorado, no obstante, la transposición didáctica es considerada un proceso y no una práctica individual. Cardelli (2004) 
critica a Chevallard, al tildar de incompleto su enfoque debido a que excluye los elementos hegemónicos e ideológicos que son constituyentes de este proceso.

Por otra parte, la acción didáctica requiere de ciertos parámetros que le permitan no solo medir, sino también contar con un panorama respecto del grado de aprendizaje y asimilación de los contenidos por parte del estudiantado. En este sentido, emerge el concepto de evaluación educativa, el cual se concibe como una herramienta que permite entender y valorar los procesos y resultados de la intervención educativa.

Referido a la evaluación, esta ha evolucionado como concepto a través del tiempo. Tal como lo destacan Ahumada (2001) y De La Garza (2004), la evaluación durante siglos estuvo asociada al juicio respecto del valor de un objeto. Acebal (2004) añade que a principios del siglo XX la evaluación es vinculada con la medición, en cuyo caso se le considera objetiva y se transforma en una medida del rendimiento académico de estudiantes. Acebal (2004) además señala que a mediados de siglo, y a partir del aporte de Tyler, la evaluación se concibe como un proceso, el cual permite determinar la brecha entre lo que se logra y lo que se desea lograr, plenamente supeditada al cumplimiento de los objetivos trazados. De acuerdo con De La Garza (2004), esta postura fue ampliamente criticada por evaluadores como Cronbach, Scriven o Atkin, quienes reaccionaron contra esta concepción y propusieron que la evaluación esté orientada principalmente a buscar información que permita tomar decisiones relativas a la enseñanza.

En esta línea, el proceso formativo debe incluir una evaluación que considere el espectro temporal: al inicio (evaluación diagnóstica), en el transcurso (evaluación formativa) y al final del desarrollo curricular (evaluación sumativa). De acuerdo con el actor que lleva a cabo la evaluación, es posible clasificarla en autoevaluación, coevaluación y heteroevaluación (Castro et al., 2004).

En la praxis evaluativa, una de las tareas más complejas es la construcción de instrumentos o procedimientos de evaluación. Según Castro et al. (2004), la correcta elaboración de un instrumento evaluativo implica cumplir las siguientes etapas:

1. Planificación: implica determinar el propósito de la evaluación, los objetivos a evaluar, el tipo de procedimiento evaluativo.

2. Construcción: implica elaborar situaciones de evaluación en un determinado formato, junto con las respectivas instrucciones y elaborar la pauta de corrección.

3. Administración: implica mantener las condiciones ambientales y de trabajo, cumplir con el tiempo asignado y aclarar potenciales dudas e interrogantes del alumnado.

4. Análisis: implica corregir de acuerdo con la pauta, tabular los resultados y determinar indicadores de logro.

5. Certificación: implica asignar puntuaciones por objetivo logrado, y convertir los resultados de logro en calificaciones.

Entre los instrumentos de evaluación se encuentran los siguientes: Listas de cotejo, registros anecdóticos, test, pruebas y cuestionarios, diarios de clase, entrevistas, análisis de casos, contrato educativo, portafolio (Castro et al., 2004). Este último constituye uno de los instrumentos evaluativos incluido con mayor frecuencia en la praxis docente actual. El uso del portafolio proviene del mundo de las artes, en particular, de la arquitectura y el diseño, el cual surge de la necesidad de demostrar competencias profesionales en el mercado laboral. Como tal, es incorporado al campo educacional como metodología de enseñanza y evaluación (Barragán, 2005).

En las últimas décadas, la praxis docente ha ido incorporando nuevas herramientas que facilitan el proceso de enseñanza-aprendizaje. Una de ellas es el denominado aprendizaje basado en problemas (ABP). El ABP o PBL (por Problem-Based Learning), de acuerdo con Barrows, es un "método de aprendizaje basado en el principio de usar problemas como punto de partida para la adquisición e integración de los nuevos conocimientos" (Morales y Landa, 2004, p.147). Este método fue desarrollado en las décadas de 1960 y 1970 en la Facultad de Ciencias de la Salud de la Universidad de McMaster, Canadá, con el fin de lograr una mejor 
preparación estudiantil para así satisfacer las demandas de la práctica profesional. Experiencias similares a esta se replicaron, en el mismo periodo, en las universidades de Michigan, Maastricht (Holanda), Newcastle (Australia), New Mexico (EEUU), Harvard y Sherbrooke (Canadá). No obstante, esta herramienta no es exitosa, si no existen las condiciones necesarias para el desarrollo del ABP. En este sentido, Comelli et al. (2002) destacan, como condiciones necesarias, el contar con alumnado activo, independiente, con autodirección en su aprendizaje y orientado a la solución del problema y no mero receptor pasivo, es decir, alumnado motivado.

El presente proyecto pretende incorporar el debate, como instrumento de evaluación, a la praxis docente del personal académico de la Facultad de Ciencias Empresariales de la Universidad del Bío-Bío. En este sentido, el debate se destaca como una herramienta pedagógica que permite desarrollar, en el estudiantado, habilidades ligadas al pensamiento crítico, al razonamiento lógico, a la comprensión de puntos de vistas opuestos, a la comunicación de ideas y al trabajo en equipo (Darby, 2007; Estaville, 1988; Pernecky, 1997). Vo y Morris (2006) conciben el debate como una forma de aprendizaje activo que permite que estudiantes migren de su estado pasivo como mero recepto de conocimiento e información. Por su parte, Ruiz (2007) plantea el debate como instrumento adecuado para la medición de habilidades relativas al manejo de la información, trabajo colaborativo, y pensamiento crítico.

En la elaboración del instrumento de evaluación, Ruiz (2007) propone que el debate debe planificarse en tres etapas:

i. Análisis de la situación: en esta etapa se deben revisar experiencias de debate previas, con el fin de identificar ciertos patrones de comportamiento en el alumnado que deben ser mejorados, así como también el lugar, físico o virtual, en el cual la actividad tendrá lugar,

ii. Establecimiento de objetivos: generalmente estos están ligados a fomentar la capacidad de investigación, el trabajo colaborativo, y la construcción de un pensamiento crítico sobre la base de antecedentes objetivos.

iii. Definición y evaluación de la actividad de debate: este instrumento permite evaluar el "saber hacer" (procedimientos) y el "saber ser" (actitudes) a través de la observación, se focaliza en elementos tales como la cantidad y calidad de la información recopilada, la cantidad y calidad de las intervenciones, y el trabajo en equipo.

Adicionalmente, la evidencia consultada (Darby, 2007; Estaville, 1988; Pernecky, 1997; Ruiz, 2007; Vo y Morris, 2006) señala que el debate debe desarrollarse respetando las siguientes fases:

1. Exposición del problema a debatir.

2. Discusión de la problemática planteada.

3. Conclusión respecto de los puntos de vistas de cada postura.

4. Elaboración del protocolo, entendiéndose este como el registro escrito tanto de la problemática discutida como de las conclusiones.

Respecto de la primera fase, Estaville (1988) señala que uno de los elementos claves de todo debate es la problemática en discusión, la que debe generar cierta controversia y ser cuidadosamente redactada para evitar cualquier confusión sobre sus alcances. Para Vo y Morris (2006) la regla general sobre el tópico a debatir es que la controversia siempre esté relacionada con los contenidos de la asignatura. En el ámbito de la asignación de tópicos, Pernecky (1997) sugiere no asignarlos al inicio del semestre, dado que esto restringe las posibilidades estudiantiles de desarrollar una posición predilecta y debatir con entusiasmo y menor ansiedad. A diferencia de Pernecky (1997), Vo y Morris (2006) proponen asignar los temas a debatir al principio del semestre para así permitir que el estudiantado se prepare el mayor tiempo posible ya que los debates son realizados al término del periodo lectivo. 
Respecto de la segunda fase, Estaville (1988) sugiere que en la ejecución del debate cada intervención debe estar respaldada por una breve cita de la fuente que avala la opinión estudiantil. Pernecky (1997) plantea una variante en la que identifica una fase constructiva-afirmativa y otra constructiva-negativa en las que las posturas a favor y en contra, respectivamente, desarrollan sus argumentos por separado y sin interrupción alguna. Por su parte, Vo y Morris (2006) añaden una fase preliminar de investigación individual en la que estudiantes deben elaborar un ensayo previo al debate.

Respecto de la tercera fase, Pernecky (1997) y Darby (2007) agregan una fase complementaria de interacción con la clase, en la que estudiantes que no participan debatiendo pueden realizar preguntas y observaciones, o bien, expresar posiciones alternativas que no fueron expuestas en el debate.

Respecto de la cuarta fase, Estaville (1988) indica que este registro debe basarse en las anotaciones tomadas por el personal docente moderador y ser individuales, al igual que la evaluación del desempeño, aún cuando el estudiantado haya sido separado en equipos. En este plano, Pernecky (1997) señala que el estudiantado prefiere la evaluación individual por sobre una grupal, aún cuando esta última genera una mayor colaboración al interior del equipo. En particular, el autor subraya que el trabajo en equipo refuerza entre sus miembros la relevancia de ser confiable y de confiar en otras personas.

Adicionalmente, la implementación del debate en el aula impone desafíos al personal docente respecto de la definición del número de instancias, el mecanismo para configurar los equipos y el tamaño de estos mismos. Referido al número de debates, Estaville (1988) plantea que su frecuencia podría estar restringida por los requerimientos de la asignatura, el tamaño del curso y el tiempo disponible. No obstante, el autor sugiere un mínimo de tres y un máximo de cinco debates durante el semestre.

Con respecto al mecanismo para configurar los equipos, existen variadas formas para distribuir estudiantes en grupos que representarán posiciones opuestas en los debates. Estaville (1988) y Pernecky (1997) plantean aplicar un cuestionario sobre un conjunto de temáticas controversiales al inicio del curso y pedirles pronunciarse respecto de su grado de acuerdo, desacuerdo o neutralidad al respecto.

Referente al tamaño de los equipos, Estaville (1988) sugiere equipos de 5 a 7 estudiantes para una sesión de 50 minutos y de 8 a 10 personas en una jornada de 75 minutos. Por el contrario, Pernecky (1997), a partir de su experiencia docente en cursos de economía, sugiere configurar los equipos con dos o tres estudiantes, ya que un número mayor entorpecería las etapas de argumentación y de refutación. En el esquema planteado por Pernecky (1997), en una sesión de 85 minutos, cada estudiante posee 8 minutos para plasmar su punto de vista en la etapa de argumentación, divide equitativamente el tiempo restante entre las etapas de refutación y de interacción con la audiencia.

Por otra parte, el éxito en la implementación del debate en el aula no depende únicamente de cuán desafiante o controversial sea la problemática en cuestión. Darby (2007) destaca como elemento clave el grado de preparación del estudiantado, tanto a nivel individual como grupal. Para Estaville (1988), el personal docente moderador es crucial en la implementación exitosa del debate, ya que, junto con promover un clima de interacción espontánea, este debe registrar las intervenciones estudiantiles, controlar el tiempo asignado, analizar la calidad y pertinencia de las opiniones y sugerir puntos no abordados que enriquezcan el debate.

Aún cuando la utilización del debate como herramienta pedagógica en la enseñanza de la economía es incipiente respecto de otras disciplinas como psicología, sociología o ciencias (Vo y Morris, 2006), la bibliografía distingue la existencia de efectos positivos tras su utilización. Al respecto, Pernecky (1997) recalca que sus estudiantes mejoran sus habilidades de investigación al consultar no solo medios de comunicación escrita sino que incluso fuentes electrónicas de estadísticas económicas actuales. Lo anterior se debe a que el autor motiva a sus estudiantes a que en la elaboración de su argumento utilicen tanto la teoría como la evidencia empírica presente en estudios académicos o documentos con estadísticas relevantes. Por su parte, Vo y Morris (2006) le atribuyen al debate la mayor comprensión de los contenidos entre el alumnado como impacto de corto plazo y el fortalecimiento del pensamiento crítico y analítico como efecto positivo de largo 
plazo. En particular, capturan la percepción de sus estudiantes a través de la aplicación de una encuesta, cuyos resultados permiten validar estadísticamente los impactos descritos atribuibles al debate.

Finalmente, cabe señalar ciertas desventajas que presenta el debate como herramienta pedagógica. Al respecto, Darby (2007) señala que ciertas temáticas pueden ser reducidas a una dualidad del tipo "blanco o negro", por lo que aquellas de carácter multidimensional podrían ser abordadas de mejor forma a través de una discusión abierta y no mediante un debate. Por otra parte, el autor indica que el sesgo competitivo del debate podría generar ansiedad y frustración en el alumnado.

\section{Metodología}

El marco metodológico del estudio involucró una serie de actividades relacionadas con el diseño, implementación y retroalimentación desde actividades de debates así como con la elaboración de productos para su replicabilidad. Estas principalmente fueron llevadas a cabo sobre la base de tres etapas: marco general y elementos centrales de la actividad; experiencia piloto y retroalimentación, y desarrollo de la actividad y elaboración de productos finales.

En una primera parte, se establece el marco general sobre la base de un conjunto de elementos clave desde los cuales se desarrolla el debate. Uno de los aspectos importantes a tener en cuenta es la asignación de los tiempos. En este sentido, la limitación del tiempo asignado para debatir genera un ambiente de presión que demanda un esfuerzo para seleccionar y focalizar la intervención en aquellos aspectos más importantes. Para el registro de los tiempos se construyó un semáforo, operado manualmente, que indica visualmente y auditivamente, los momentos de inicio, transcurso y finalización de cada intervención.

Otro componente clave importante para reforzar el carácter formal de la actividad fue la participación de docentes. En este contexto, se conformó un jurado constituido por 3 personas, en el cual se incluyó al profesor o profesora responsable del curso.

Adicionalmente, con el objeto de estimular una preparación adecuada del alumnado, se hizo uso de incentivos sobre la base de premios y sanciones. En este sentido, los incentivos tuvieron un carácter individual y grupal, este último premia el carácter colaborativo de la actividad. En el caso de los incentivos individuales, se entregaron a un número limitado de estudiantes sobresalientes, independientemente si pertenecían al equipo ganador o perdedor. Estos correspondían a puntos adicionales para alguna otra evaluación tradicional o una nota individual superior a la nota promedio que obtuvo su equipo. En relación con los incentivos grupales, se entregaron puntos adicionales para cada miembro del equipo para alguna otra evaluación tradicional. Además, la evaluación grupal del equipo ganador fue superior a la calificación obtenida por el grupo perdedor. Conjuntamente, con el objeto de incentivar el cumplimiento de los tiempos, se aplicaron sanciones para el grupo que excedió el tiempo asignado. Se descontaron puntos a todo el grupo por cada uno de sus miembros que excedieron los tiempos estipulados en el protocolo.

En una segunda etapa, siguiendo la revisión de la bibliografía (Darby, 2007; Estaville, 1988; Vo y Morris, 2006), se confeccionó un instrumento borrador para desarrollar una experiencia piloto o predebate, con la pauta y hoja de evaluación, protocolo de la actividad e indicaciones generales. Se elaboró un instrumento con versiones alternativas que se adapta a la realidad de cada asignatura, en función de sus contenidos y tamaño del curso. Se seleccionó una muestra de cursos del área de economía para aplicar este instrumento. Con el objeto de que la actividad tenga un carácter formal, su evaluación fue incorporada dentro del proceso de evaluación de la asignatura o en su defecto se ofrecieron incentivos sobre la base de puntos para alguna evaluación escrita. Esta etapa involucró el procesamiento de los resultados, la evaluación de la actividad y comunicación de los resultados a estudiantes. Lo anterior permitió recoger percepciones y recomendaciones de estudiantes y docentes participantes en el proceso de evaluación.

En una tercera etapa, se realizó una revisión del instrumento y se incorporaron las modificaciones sugeridas. Se ajustó el procedimiento del debate y se recomendaron protocolos alternativos que consideran algunas 
características del curso. Se discutieron los criterios de evaluación: se validaron algunos de ellos, se descartaron otros y se incluyeron algunos nuevos. El instrumento ajustado por las recomendaciones surgidas durante la experiencia piloto fue aplicado nuevamente a un conjunto de asignaturas del área de economía. En estas actividades se utilizó el equipamiento definitivo, incluido el semáforo. La actividad se desarrolló en función del protocolo ajustado. Se recogieron los comentarios y sugerencias de parte del jurado y alumnado. Finalmente, se incorporaron las sugerencias, y se realizaron las modificaciones finales para los protocolos, criterios de evaluación y procedimientos generales; de esta manera se obtuvieron los productos definitivos.

\section{Resultados}

La metodología de trabajo exige la aplicación preliminar de los instrumentos en experiencias piloto que otorguen cierta flexibilidad para poder introducir modificaciones y versiones alternativas. En esta primera parte, no se utiliza equipamiento y los cursos son seleccionados en función de la carga académica y la disponibilidad de docentes. Una vez ajustados los instrumentos con las sugerencias y recomendaciones surgidas durante la experiencia piloto, se desarrollan experiencias formales de debate, para lo cual se seleccionan los cursos del área de economía que mejor se ajustan a la dinámica de discusión que se requiere en este tipo de actividades.

\section{Experiencia piloto}

La experiencia piloto se llevó a cabo durante el primer semestre de 2009. Para ello se contó con la colaboración de estudiantes de las asignaturas Introducción a la Microeconomía, dictada en la carrera de Ingeniería Comercial, y las asignaturas Economía II y Comercio Exterior, ofrecidas en la carrera de Contador Público y Auditor. El jurado estuvo constituido por el personal docente responsable de las asignaturas, César Salazar y Cristián Pinto y los docentes Andrés Acuña y Gabriel Toro, todos ellos académicos del Departamento de Economía y Finanzas de la Facultad de Ciencias Empresariales. Cabe señalar que, en cada instancia de debate, el jurado se encontraba frente al estudiantado en el momento en que este defendía sus posturas. En este ámbito, Pernecky (1997) propone tomar notas en un lugar alejado de estudiantes que presentan sus argumentos y contraargumentos, para así reducir el contacto visual inhibidor de su desempeño y su ansiedad.

Para el caso del curso de Introducción a la Microeconomía, se propuso un debate tipo mesa redonda. El tema a debatir fue "Control de precios en la agricultura". El profesor responsable fue quien dirigió y evaluó la actividad. La metodología comprendió, en una primera ronda, la exposición individual de cada estudiante. Tal como lo destacan Estaville (1988), Pernecky (1997) y Vo y Morris (2006), esta primera ronda exige a cada estudiante destinar tiempo para investigar sobre los alcances de la controversia planteada para así elaborar un argumento coherente. Desde esta intervención, quien moderaba desprendió la posición en contra o a favor de cada estudiante. Hubo alumnado indeciso. Por esta razón, se realizó una segunda ronda donde el alumnado defensor de cada una de las posturas trataba de convencer a quienes mostraban indecisión para lograr su alianza. Los equipos se situaron uno frente al otro. Esta ronda fue dirigida por 2 representantes de cada quipo. De esta manera se formaron los grupos constituidos por estudiantes en contra y a favor. Luego se desarrollaron dos rondas adicionales con la utilización de un protocolo preliminar y un conjunto de criterios de evaluación previos. En estas instancias, la representación de cada equipo también estuvo a cargo de dos estudiantes.

En general, la actividad se desarrolló normalmente sin mayores inconvenientes. Sin embargo, debido a que el alumnado no tenía experiencias previas similares, se percibió cierto recelo e incertidumbre en relación con esta actividad, situación que generó la inasistencia e impuntualidad de un número importante y falta de dinamismo durante el comienzo. Esto, además, pudo ser causa de la estructura de la mesa redonda que exigía 
algún nivel de improvisación y sorpresa. En la medida en que la actividad fue transcurriendo, el alumnado se fue convenciendo e involucrando, lo que motivó una interesante discusión. Finalmente, el grupo en contra fue el vencedor. La evaluación de la actividad constituyó un $10 \%$ de la nota final del curso. El $50 \%$ de la nota del debate correspondió a una evaluación que consideró la intervención individual de la primera ronda. El 50\% restante consideró la evaluación de cada postura. Se entregaron 5 puntos por el certamen para cada miembro del equipo ganador y 5 puntos adicionales para quienes destacaran en su intervención individual en la primera ronda. La evaluación del estudiantado fue muy positiva, situación que motivó por su parte la solicitud de repetir la actividad, lo cual por motivos de tiempo no pudo ser posible.

Un segundo debate fue realizado en la asignatura Comercio Exterior de la carrera Contador Público y Auditor. El tema a debatir fue: "Crisis económica global: ¿Es deseable que el gobierno intervenga restringiendo las condiciones para el comercio internacional?". El jurado evaluador estuvo compuesto por tres docentes dentro de los cuales se encontraba el profesor responsable de la asignatura.

Considerando el número de estudiantes del curso, el cual superaba las 50 personas, se decidió modificar el formato para facilitar el desarrollo de la actividad. De esta manera, la evaluación contempló la formación de grupos de 3 o 4, quienes mediante un trabajo colaborativo desarrollaron un informe donde exponían su visión a favor o en contra de la pregunta. Es así como cada grupo debía pronunciarse sobre defender la posición a favor o en contra, a través del desarrollo de un ensayo donde presentaban sus argumentos de manera escrita. Este requerimiento, previo a la realización del debate, es similar al descrito por Estaville (1988) en su experiencia con estudiantes de geografía de Clemson University, o por Vo y Morris (2006) en su implementación del debate en cursos introductorios de economía en Winthrop University, ambas universidades situadas en Estados Unidos. Una vez entregado este documento, el profesor procedió a separar a los grupos en función de su postura, esto debido a que, según Pernecky (1997), el estudiantado exhibe un mayor compromiso y fervor si debate defendiendo su posición personal.

Tras la entrega del ensayo, hubo un tiempo de preparación para el desarrollo de la actividad presencial, en la cual el alumnado debía comunicar sus ideas y fundamentos para defender cada posición. Para ello, en una primera ronda, cada grupo elegía su representante o líder para presentar su visión del tema y defender su postura. Es decir, esta primera ronda es coherente con las fases constructiva-afirmativa y constructivanegativa descritas por Pernecky (1997) en su experiencia con estudiantes de economía en Saint Olaf College, Estados Unidos. Un elemento no explorado en esta etapa fue haberle otorgado al líder la responsabilidad de resumir en la forma de un breve reporte o ensayo los aportes de miembros de su equipo, tal como se plantea en la experiencia docente descrita por Vo y Morris (2006). Cabe destacar que esta etapa se desarrolló de forma normal, sin mucha dinámica, puesto que el alumnado se centró en exponer sus argumentos con base en los contenidos de su informe.

Siguiendo la recomendación de Pernecky (1997), tras la primera ronda se realizó una pausa de cinco minutos. De acuerdo con el autor, una pausa extensa desincentivaría una mayor preparación de los equipos, reduciría la continuidad del debate y lo tornaría tedioso para el público presente.

Posteriormente, en una segunda etapa se solicitó al alumnado presentar nuevamente sus argumentos, tras una breve reorganización de los grupos. En esta segunda intervención, se percibió un poco más de dinamismo e interacción. Finalmente cada posición cerró el debate con la exposición de un representante. Cada presentación, desde la primera etapa, fue evaluada y su calificación se ponderó en las actividades de evaluación del curso. El grupo que destacó en su presentación obtuvo 10 puntos adicionales para el certamen. Todo el alumnado que defendió la posición ganadora recibió 2 puntos adicionales para el certamen.

La experiencia demostró que es posible estructurar una dinámica de aula de tal manera que se configure un procedimiento para lograr que la totalidad de estudiantes participe, a pesar del tamaño excesivo de los cursos. Sin embargo, claramente, mientras se incrementa el número de participantes se dificulta la logística de la actividad, y se pierde cierto nivel de dinamismo. Asimismo, se presume que la pasividad con que se llevó a 
cabo la actividad obedece a la rigidez con que se condujo el debate y a la falta de comunicación de los objetivos que se perseguían durante cada ronda.

Una tercera experiencia se desarrolló para el caso de la asignatura Economía II, dictada en la carrera de Contador Público y Auditor. El curso era bastante numeroso, por lo cual se decidió realizar dos debates por separado con temas distintos. El primero de ellos discutía la "Propuesta de intervención del gobierno en empresas con riesgo de quiebra", mientras que el segundo tema trataba de las "Propuestas de flexibilidad en el mercado del trabajo". La separación del curso permitió definir un número reducido de participantes, lo que facilitó la logística y la ejecución de la actividad. Esta definición facilitó la intervención de todo el alumnado tanto en la primera como en la segunda ronda.

El primer grupo fue evaluado por tres profesores y el segundo por solamente dos. Se observó cierta heterogeneidad en la calidad de las intervenciones lo que dificultó la evaluación con criterios de trabajo en equipo. En esta experiencia, como en el resto de las actividades, la evaluación se realizó de manera individual y la calificación de cada grupo se obtuvo como el promedio de cada quien de sus integrantes. Las diferencias en la calificación final entre secciones generó cierto descontento de una parte del alumnado, quienes cuestionaron la forma de evaluación. El grupo de menor rendimiento presentó su desacuerdo, cuestionando la elección de solo dos jurados para la actividad, en vez de tres profesores, como en el caso de la otra sección. Particularmente en este curso, se flexibilizaron los tiempos para facilitar la expresión de ideas de estudiantes con discapacidad en el habla. Se otorgaron 5 puntos para el certamen para cada miembro del equipo ganador. Se premió individualmente a dos que hubieran destacado en la exposición de cada equipo con 5 puntos adicionales. La actividad fue evaluada con una ponderación del $10 \%$ en el promedio final del curso.

\section{Experiencia formal}

Durante el segundo semestre de 2009, se desarrollaron tres experiencias formales de debate, en las cuales se hizo uso del equipamiento e instrumentos ajustados de acuerdo con la experiencia piloto. Los cursos elegidos fueron Introducción a la Macroeconomía y Economía Internacional, ambos ofrecidos en la carrera de Ingeniería Comercial en el segundo y cuarto año del plan de estudios, respectivamente. Se contó con la colaboración de 5 integrantes del personal académico del Departamento de Economía y Finanzas: Andrea King (directora de Escuela de la carrera de Ingeniería Comercial), Gabriel Toro (director del Departamento de Economía y Finanzas), Osvaldo Pino, Marcela Bertín y Cristián Pinto. Se aplicó un formato estándar, como el presentado en el Anexo 1, 2, 3, 4, 5, 5b).

El curso de Economía Internacional comprendía dos secciones, cada una de ellas con un número aproximado de 30 estudiantes. Esta situación dificultó la participación de la totalidad del alumnado en cada una de las rondas del debate. Con el objeto de facilitar la logística y la dinámica de la actividad, se decidió para la segunda ronda limitar la participación a solo 5 de cada equipo. En la ronda final, cada equipo elegía a un representante para cerrar la actividad. En esta oportunidad, considerando que en una de las secciones existía un número similar de alumnos de sexo masculino y femenino, se decidió separar los equipos tomando en consideración este criterio. Para el segundo caso, no se cumplían estas condiciones, por lo que la asignación de los grupos se hizo de manera aleatoria.

Los temas a debatir fueron: "Estrategia de inserción internacional: Priorizar relaciones comerciales con naciones vecinas del continente o priorizar relaciones comerciales con naciones desarrolladas"; y "Políticas comerciales en periodos de crisis: Se deben definir medidas para proteger la industria nacional en períodos de inestabilidad internacional o la inestabilidad internacional que se origina en períodos de crisis es parte de las reglas del juego del comercio internacional, por lo que no se justifican las medidas de protección”. La elección de estas temáticas siguió el criterio propuesto por Vo y Morris (2006), quienes sugieren que, al momento de elegir la controversia a debatir, esta se vincule con los contenidos de la asignatura y que, además, la selección 
se centre en el estudiantado, pues debe considerar el probable grado de entendimiento de los alcances de la problemática por su parte. Ambas experiencias se desarrollaron satisfactoriamente.

Para el caso donde los equipos fueron definidos según el género, la actividad fue mejor evaluada. Se presume que la organización de los equipos con base en criterios que permitan definir grupos homogéneos podría profundizar sentidos de pertenencia e identidad que motiven una mejor comunicación y organización en torno a la defensa de una idea común. En contraste, se observaron algunos problemas de organización para el caso de la actividad donde los grupos fueron definidos aleatoriamente. En esta experiencia se pudo constatar una muy baja relación entre integrantes de cada equipo ${ }^{[4]}$, situación que podría explicar la falta de dinamismo con que se desarrolló la actividad. Se premió al equipo ganador con 5 puntos para el certamen y a quienes mejor expusieron de cada equipo con 5 puntos adicionales. La evaluación contempló un $10 \%$ de la nota final del curso.

Desde esta experiencia surgió la necesidad de considerar el derecho a réplica por lo menos durante la última ronda del debate. El alumnado manifestó la necesidad de contar con la oportunidad de contra-argumentar. Además, se sugiere evaluar las rondas con criterios distintos. Se argumenta que en la primera ronda, debe prevalecer una evaluación de carácter individual, en donde cada persona expositora sea sometida a una serie de criterios que obedecen a una preocupación y desempeño personal, como por ejemplo, presentación personal, primera impresión, imagen, etc. Luego, durante la segunda ronda, se sugiere definir criterios de evaluación de carácter grupal, como por ejemplo, organización, debate de ideas, etc.

Una experiencia similar, con el mismo formato, se desarrolló para la asignatura Introducción a la Macroeconomía. El tema a debatir fue "Indexación de salarios en UF", cuya selección se basó en el criterio descrito previamente. Se separaron los grupos entre hombres y mujeres. El jurado fue constituido por dos personas y se entregaron los mismos incentivos que en el caso de Economía Internacional. La asignatura se ofrece durante el segundo año de la carrera, situación que garantiza un mayor grado de homogeneidad del curso. La experiencia fue muy satisfactoria, a pesar de algunas deficiencias conceptuales detectadas propias de estudiantes de los primeros años. Este hecho hace necesario considerar el estado de avance estudiantil dentro de la línea de economía del plan de estudios en la definición de las exigencias y expectativas sobre el desarrollo de la actividad. En este sentido, se podría acoger la sugerencia de Pernecky (1997) de entregar material de apoyo al estudiantado respecto del tópico a debatir. Aún cuando esto puede desincentivar el ímpetu y responsabilidad del alumnado por investigar fuentes de información de manera autónoma, el autor considera que esta práctica es recomendable en cursos introductorios.

Finalmente, cabe destacar que tanto en la experiencia con Economía Internacional como Introducción a la Macroeconomía, el uso del semáforo cumplió las expectativas, al trasformarse en un mecanismo de presión durante las exposiciones y contribuir a cumplir los tiempos de la actividad.

\section{CONCLUSIONES}

Los debates constituyen un instrumento de aprendizaje activo que se ajusta a la dinámica de la discusión presente en temas económicos, su utilización es incipiente en la enseñanza de esta ciencia social respecto de otras disciplinas (Vo y Morris, 2006). En este sentido, proporciona una oportunidad para que el alumnado desarrolle el razonamiento lógico y el pensamiento crítico, refuerce su capacidad de expresión y opinión frente a los acontecimientos que afectan su entorno, fortalezca su autoestima y fomente su creatividad (Darby, 2007; Estaville, 1988; Pernecky, 1997; Vo y Morris, 2006). Lo anterior se encuentra en sintonía con los cambios observados en el paradigma de la práctica docente, y en el modelo educativo que están proponiendo las universidades, el cual centra su atención en el alumnado. Este trabajo propone un diseño, implementación y sistematización de experiencias de actividades de debate en economía. Las actividades se llevaron a cabo con estudiantes de programas ofrecidos por la Facultad de Ciencias Empresariales de la Universidad del BíoBío, campus Concepción, Chile. 
Una de las principales conclusiones de este estudio es que la motivación resulta fundamental para el desarrollo exitoso de esta actividad, pues incide positivamente en el grado de preparación estudiantil al momento de enfrentar el debate (Darby, 2007). Al respecto, tal como lo destaca Estaville (1988), el rol del profesorado es vital para hacer sentir a sus estudiantes la importancia del debate, dándole el realce que se merece, para generar cierta motivación previa. Para ello, se concluye que es importante hacer pública la actividad. Asimismo, se destaca la importancia de comunicar correctamente los detalles de la actividad y aclarar todas las inquietudes de los procedimientos y reglas previo al inicio del debate, tal como lo remarcan Estaville (1988) y Vo y Morris (2006). Sin embargo, se sugiere mantener cierto grado de flexibilidad para realizar cambios sobre la marcha, para permitir intervenir en función de sucesos inesperados que podrían amenazar el éxito del debate. Por esta razón es importante dar cierto espacio a la improvisación cuando esta se requiera, siempre y cuando no se atente con el cumplimiento de las reglas y procedimientos definidos en el protocolo.

Desde las experiencias de debate, se recomienda definir los equipos intencionadamente en función de criterios que creen cierto grado de identidad y compromiso, tal como lo sugiere Pernecky (1997). Una posibilidad podría ser dividir el curso entre hombres y mujeres, por generación, etc. Esto es importante, especialmente en cursos superiores donde el alumnado proviene desde distintas generaciones debido a su dispar avance en el currículo de su carrera profesional.

En la evaluación de la actividad, se recomienda la participación no solo de cada docente responsable de la asignatura, sino además de más personal académico del área. Si la división de los grupos se realizó según el género, se sugiere integrar en el jurado a profesoras. Además, es vital el apoyo logístico de una persona ayudante para el control de los tiempos y la manipulación del semáforo.

Debido a restricciones de tiempo y espacio físico, así como complejidades logísticas para el desarrollo de la actividad, se recomienda su realización con un número de no más de diez estudiantes por equipo. Esto es coherente con el número de integrantes propuesto por Estaville (1988), al considerar que cada bloque de docencia tiene una extensión de 80 minutos en la Universidad del Bío-Bío. No obstante, es muy difícil que estas condiciones óptimas se cumplan.

Esta experiencia demuestra que es posible el diseño e implementaciones de protocolos alternativos, con ciertas variaciones a la actividad. En este trabajo se presentan algunas versiones alternativas como mesas redondas y debates grupales que se ajustan a cursos numerosos. En particular, la variante de mesa redonda a través de una discusión abierta permitiría abordar temáticas de carácter multidimensional que no se ajustan al formato tradicional del debate (Darby, 2007).

Con el objeto de promover esfuerzos individuales y de equipo por la parte estudiantil, se concluye que es fundamental otorgar premios y sanciones para quienes ganen y para quienes no respeten las reglas, respectivamente. En relación con los incentivos individuales, dado que en general los protocolos de los debates recomiendan el cierre de este con la intervención de un o una representante por cada equipo, este reconocimiento lo realiza el mismo alumnado cuando decide voluntariamente quien lo representará en esta instancia final. Estos incentivos pueden tomar la forma de puntos para alguna evaluación tradicional. Sin embargo, la evaluación final debe ser única para la totalidad de integrantes de un mismo equipo, diferenciándose solo para quienes obtuvieron algún reconocimiento.

Con el fin de retroalimentar a los grupos de estudiantes y de esta manera contribuir a su proceso de aprendizaje, es primordial que cada docente responsable de la asignatura concluya el debate destacando los aspectos positivos y negativos de cada grupo, así como resaltando los argumentos más destacados a favor y en contra de cada postura.

Por otra parte, una vertiente de investigación futura sería aplicar una encuesta a estudiantes en el formato y alcances propuestos por Vo y Morris (2006). Lo anterior permitiría contar con evidencia cuantitativa respecto del grado en que el alumnado percibe los beneficios de corto y largo plazo en su formación profesional tras experimentar el debate en el aula. 
Finalmente, para que los resultados sean los esperados, se requiere que este tipo de actividades se desarrollen de manera sistemática durante todo el proceso de formación. Por tal motivo es necesario comprometer la participación activa del personal académico y precisar explícitamente el "debate" como una actividad formal de evaluación en los programas de las asignaturas.

\section{ReFERENCIAS}

Acebal, A. M. (2004). Aspectos a ser tenidos en cuenta en un plan de evaluación de la calidad educativa a nivel nacional. Revista Iberoamericana de Educación, 35(2), 1-12.

Ahumada, P. (2001). La evaluación en una concepción de aprendizaje significativo. Valparaíso: Ediciones Universitarias de Valparaíso.

Azócar, R. E. (Enero-marzo, 2006). Reflexiones epistemológicas corriente racionalista-deductiva. Episteme, 7.

Barragán, R. (2005). El portafolio, metodología de evaluación y aprendizaje de cara al nuevo espacio europeo de educación superior. Una experiencia práctica en la Universidad de Sevilla. Revista Latinoamericana de Tecnología Educativa, 4(1), 121-140.

Cardelli, J. (2004). Reflexiones críticas sobre el concepto de transposición didáctica de Chevallard. Cuadernos de Antropología Social, 19, 49-61.

Castro, F., Correa, M. E., y Lira, H. (2004). Curriculum y evaluación - Texto guía. Chillán: Universidad del Bío-Bío. Chevallard, Y. (1991). La transposición didáctica: Del saber sabio al saber enseñado (2a ed.). Buenos Aires: AIQUE.

Comelli, N., Ortiz, E., y López, M. (2002). El aprendizaje basado en problemas. Una propuesta didáctica para el área fisicoquímica. Congreso Regional de Ciencia y Tecnología NOA 2002, Universidad Nacional de Catamarca.

Darby, M. (2007). Debate: A teaching-learning strategy for developing competence in communication and critical thinking. Journal of Dental Hygiene, 81(4), 1-10.

De La Garza, E. L. (2004). La evaluación educativa. Revista Mexicana de Investigación Educativa, 9(23), 807-816.

Estaville, Jr., L. E. (1988). Debate: A teaching strategy for geography. Journal of Geography, 87(1), 2-4.

Facultad de Ciencias Empresariales. (2008). Proyecto de reforma del plan de estudios de la carrera de Ingeniería Comercial. Facultad de Ciencias Empresariales, Universidad del Bío-Bío, Chile.

Morales, P., y Landa, V. (2004). Aprendizaje basado en problemas. Theoria, 13, 145-157.

Pernecky, M. (1997). Debate for the economics class-and others. College Teaching, 45(4), 136-138. doi:10.1080/87567559709596215

Ruiz, M.E. (2007). El debate como instrumento de evaluación. Departamento de Comercialización e Investigación de Mercados, Universidad de Valencia, España.

Universidad del Bío-Bío (2008). Modelo Educativo de la Universidad del Bío-Bío. Vicerrectoría Académica, Universidad del Bío-Bío, Chile.

Vo, H.X., y Morris, R.L. (2006). Debate as a tool in teaching economics: Rationale, technique, and some evidence. Journal of Education for Business, 81(6), 315-320. doi: 10.3200/JOEB.81.6.315-320

Zufiaurre, B., y Gabari, M.I. (2001). Didáctica para maestras, (2 ed.). Madrid: Editorial CCS. 


\section{Anexo 1}

\section{Protocolo debate versión estándar}

\section{Introducción}

Se hace una pequeña presentación del tema a debatir, de los grupos, del jurado, de la persona moderadora, así como de la encargada de fijar los tiempos. Se realiza una breve presentación del desarrollo de la actividad, recordando los tiempos, las sanciones, y la dinámica de cada ronda. Se sortea el equipo que comienza a exponer.

\section{Primera ronda}

Cada participante cuenta con 2 minutos para presentar argumentos en favor de su postura, y no se debe exceder dicho tiempo. En el momento de la presentación, se van alternando participantes de cada equipo. Los fundamentos presentados deben tener un carácter argumentativo, sobre la base de elementos técnicos y de evidencia empírica que avalen cada intervención. Esta es una etapa donde se deben presentar la mayor cantidad de antecedentes distintos posibles a favor de cada postura. Se sugiere que cada equipo tome nota de los expuesto por el equipo contrincante para poder utilizar estos argumentos a su favor en una segunda ronda. Sin embargo, se deja la libertad a sus participantes para comenzar a debatir en el momento que lo estimen conveniente.

Se encenderá la luz amarilla del semáforo cuando falten 30 segundos para que concluya el tiempo de cada exposición. Al finalizar el tiempo se encenderá la luz roja y sonará la chicharra. Si se hace caso omiso al llamado, se penalizará con 5 puntos a todo el equipo por cada oportunidad en que cada miembro haya excedido los tiempos estipulados.

\section{Pausa (5 minutos)}

Tiene como objetivo dar un espacio a participantes para que se preparen para la segunda ronda.

\section{Segunda ronda}

Cada participante cuenta con 2 minutos para presentar argumentos en favor de su postura, y no se debe exceder dicho tiempo. En esta etapa deberá comenzar a debatir con base en los antecedentes puestos en discusión durante la primera etapa. Esta etapa es de carácter conclusivo, y su objetivo es presentar los argumentos más sólidos para convencer a la audiencia con respecto a la postura a defender. Para tal efecto, se deben utilizar los argumentos del equipo contrario a favor de la postura defendida. Es posible utilizar como estrategia la ironía y la reducción al absurdo, siempre en un clima de formalidad y respeto por sus pares.

Se encenderá la luz amarilla del semáforo cuando falten 30 segundos para que concluya el tiempo de cada exposición. Al finalizar el tiempo se encenderá la luz roja y sonará la chicharra. Si el estudiante o la estudiante hace caso omiso al llamado, se penalizará con 5 puntos a todo el equipo por cada oportunidad que cada miembros se haya excedido en los tiempos estipulados. 


\section{Pausa (5 minutos)}

Tiene como objetivo dar un espacio a participantes para que se organicen y decidan quién va a cerrar el debate a través de sus conclusiones.

\section{Ronda final}

Cada uno de los equipos deberá elegir a su representante para que intervenga en la etapa final. Se presenta públicamente a integrantes que van a cerrar el debate. Cada quien tendrá un tiempo máximo de 2 minutos para resumir los antecedentes presentados a favor de su postura y exponer las principales conclusiones de su equipo.

Se encenderá la luz amarilla del semáforo cuando falten 30 segundos para que concluya el tiempo de cada exposición. Al finalizar el tiempo se encenderá la luz roja y sonará la chicharra. Si el estudiante o la estudiante hace caso omiso al llamado, se penalizará con 5 puntos a todo el equipo por cada oportunidad en que cada miembro haya excedido los tiempos estipulados.

\section{Pausa (5 Minutos)}

Tiene como objetivo dar un espacio para que el jurado delibere con respecto a cuál es el equipo ganador, teniendo en consideración los parámetros de evaluación de cada ronda del debate

\section{Deliberación}

El presidente o presidenta del jurado realiza una breve conclusión de los principales elementos que fueron discutidos durante la actividad, tratando de definir algunos consensos y plantear temas que aún contribuyen al debate nacional. Posteriormente, comunica el veredicto final y argumenta brevemente su decisión.

\section{Anexo 2}

\section{Protocolo debate versión mesa redonda}

\section{Introducción}

Se hace una pequeña presentación del tema a debatir, de los grupos, del jurado, de la persona moderadora, así como de la persona encargada de fijar los tiempos. Se realiza una breve presentación del desarrollo de la actividad, recordando los tiempos, las sanciones, y la dinámica de cada ronda. Se ordena en orden alfabético al alumnado desde la derecha del profesor o profesora, formando una especie de mesa redonda.

\section{Primera ronda}

Cada participante cuenta con 2 minutos para presentar argumentos en favor o en contra del tema a debatir, y no se debe exceder dicho tiempo. Los fundamentos presentados deben tener un carácter argumentativo, sobre la base de elementos técnicos y de evidencia empírica que avalen cada intervención. Esta es una etapa donde 
se deben presentar la mayor cantidad de antecedentes distintos posibles a favor o en contra de cada postura. Al finalizar su intervención el alumnado debe reafirmar su posición a favor o en contra del tema a debatir.

Se encenderá la luz amarilla del semáforo cuando falten 30 segundos para que concluya el tiempo de cada exposición. Al finalizar el tiempo se encenderá la luz roja y sonará la chicharra. Si el estudiante o la estudiante hace caso omiso al llamado, se penalizará con 5 puntos a todo el equipo por cada oportunidad en que cada miembro haya excedido los tiempos estipulados.

\section{Pausa (5 minutos)}

Tiene como objetivo dar un espacio a participantes para que se preparen para la segunda ronda y conformen los grupos en función de su postura a favor o en contra. Cada equipo debe organizarse y ubicarse frente al equipo contrario. En el caso que exista alumnado indeciso, se realizará una ronda adicional donde cada equipo tendrá 2 minutos para exponer sus ideas y convencer a sus pares para que se integren a la defensa de su postura.

\section{Segunda ronda}

Una vez formado los equipos, sus miembros tendrán en total 4 minutos para presentar argumentos en favor de su postura, y no se debe exceder dicho tiempo. Adicionalmente, cada equipo tendrá un derecho a réplica de 2 minutos en total. En esta etapa el alumnado deberá comenzar a debatir con base en los antecedentes puestos en discusión durante la primera etapa. Esta etapa es de carácter conclusivo, y su objetivo es presentar los argumentos más sólidos para convencer a la audiencia con respecto a la postura a defender. Para tal efecto, se deben utilizar los argumentos del equipo contrario a favor de la postura defendida. Es posible utilizar como estrategia la ironía y la reducción al absurdo, siempre en un clima de formalidad y respeto por sus pares.

Se encenderá la luz amarilla del semáforo cuando falten 30 segundos para que concluya el tiempo de cada exposición. Al finalizar el tiempo se encenderá la luz roja y sonará la chicharra. Si el estudiante o la estudiante hace caso omiso al llamado, se penalizará con 5 puntos a todo el equipo por cada oportunidad en que cada miembro haya excedido los tiempos estipulados.

\section{Pausa ( 5 minutos)}

Tiene como objetivo dar un espacio a participantes para que se organicen y elijan a su representante, quien debe cerrar el debate a través de sus conclusiones.

\section{Ronda final}

Cada uno de los equipos deberá elegir a su representante, quien debe pasar adelante y exponer en la etapa final. Se presenta públicamente a su integrante que va a cerrar el debate. Cada quien tendrá un tiempo máximo de 2 minutos para resumir los antecedentes presentados a favor de su postura y exponer las principales conclusiones de su equipo. Se encenderá la luz amarilla del semáforo cuando falten 15 segundos para que concluya el tiempo de cada exposición. Al finalizar el tiempo se encenderá la luz roja y sonará la chicharra. Si el estudiante o la estudiante hace caso omiso al llamado, se penalizará con 5 puntos a todo el equipo por cada oportunidad en que cada miembro haya excedido los tiempos estipulados. 


\section{Pausa (5 Minutos)}

Tiene como objetivo dar un espacio para que el jurado delibere con respecto a cuál es el equipo ganador, teniendo en consideración los parámetros de evaluación de cada ronda del debate

\section{Deliberación}

Quien preside el jurado realiza una breve conclusión de los principales elementos que fueron discutidos durante la actividad, tratando de definir algunos consensos y plantear temas que aún contribuyen al debate nacional. Posteriormente, comunica el veredicto final y argumenta brevemente su decisión.

\section{Anexo 3}

\section{Protocolo debate versión equipos}

\section{A. Primera etapa: Ensayo}

Esta primera parte involucra escribir un ensayo que no debe exceder las 2 páginas, con letra 12 Times New Roman, interlineado 1,5 y márgenes de $3 \mathrm{cms}$. El trabajo debe ser realizado por un número de 3 alumnos. En este informe, cada grupo deberá presentar una pequeña discusión sobre la base de argumentos a favor y en contra del tema a debatir. Finalmente como conclusión cada equipo deberá manifestar explícitamente su posición en contra o favor del tema en discusión.

\section{B. Segunda Etapa: Debate}

\section{Introducción}

Se hace una pequeña presentación del tema a debatir, de los grupos, del jurado, del moderador, así como de la persona encargada de fijar los tiempos. Se realiza una breve presentación del desarrollo de la actividad, recordando los tiempos, las sanciones, y la dinámica de cada ronda.

\section{Primera ronda}

Cada uno de los grupos deberá elegir a su representante, quien dispondrá de 2 minutos para presentar los aspectos mas relevantes de su ensayo, y no se debe exceder dicho tiempo. Los fundamentos presentados deben tener un carácter argumentativo, sobre la base de elementos técnicos y de evidencia empírica que avalen cada intervención. Esta es una etapa donde se deben presentar la mayor cantidad de antecedentes distintos posibles a favor o en contra de cada postura. Al finalizar su intervención el alumnado debe reafirmar su posición a favor o en contra del tema a debatir.

Se encenderá la luz amarilla del semáforo cuando falten 30 segundos para que concluya el tiempo de cada exposición. Al finalizar el tiempo se encenderá la luz roja y sonará la chicharra. Si el estudiante o la estudiante hace caso omiso al llamado, se penalizará con 5 puntos a todo el equipo por cada oportunidad en que cada miembro haya excedido los tiempos estipulados. 


\section{Pausa (5 minutos)}

Tiene como objetivo dar un espacio a sus participantes para que se preparen para la segunda ronda y conformen los equipos. De esta manera, cada equipo estará formado por aquellos grupos de estudiantes que manifestaron en forma escrita y durante la primera ronda su posición en contra o a favor del tema a debatir. Nuevamente le corresponde a cada grupo elegir a su representante para exponga en esta segunda ronda.

\section{Segunda ronda}

Cada participante cuenta con 2 minutos para presentar argumentos en favor de su postura, y no se debe exceder dicho tiempo. En el momento de la presentación, se va alternando un o una participante de cada equipo. Los fundamentos presentados deben tener un carácter argumentativo, sobre la base de elementos técnicos y de evidencia empírica que avalen cada intervención. Esta es una etapa donde se deben presentar la mayor cantidad de antecedentes distintos posibles a favor de cada postura. Se sugiere que cada equipo tome nota de los expuesto por el equipo contrincante para poder utilizar estos argumentos a su favor en una segunda ronda. Sin embargo, se deja la libertad a los participantes para comenzar a debatir en el momento que lo estimen conveniente.

Se encenderá la luz amarilla del semáforo cuando falten 30 segundos para que concluya el tiempo de cada exposición. Al finalizar el tiempo se encenderá la luz roja y sonará la chicharra. Si el estudiante o la estudiante hace caso omiso al llamado, se penalizará con 5 puntos a todo el equipo por cada oportunidad en que cada miembro haya excedido los tiempos estipulados.

\section{Pausa (5 minutos)}

Tiene como objetivo dar un espacio a sus participantes para que se organicen y elijan a un o una representante, quien debe cerrar el debate a través de sus conclusiones.

\section{Ronda final}

Cada uno de los equipos deberá elegir a su representante para que intervenga en la etapa final. Se presenta públicamente a la persona integrante que va a cerrar el debate, quien tendrá un tiempo máximo de 2 minutos para resumir los antecedentes presentados a favor de su postura y exponer las principales conclusiones de su equipo.

Se encenderá la luz amarilla del semáforo cuando falten 15 segundos para que concluya el tiempo de cada exposición. Al finalizar el tiempo se encenderá la luz roja y sonará la chicharra. Si el estudiante o la estudiante hace caso omiso al llamado, se penalizará con 5 puntos a todo el equipo por cada oportunidad en que cada miembro haya excedido los tiempos estipulados.

\section{Pausa (5 Minutos)}

Tiene como objetivo dar un espacio para que el jurado delibere con respecto a cuál es el equipo ganador, teniendo en consideración los parámetros de evaluación de cada ronda del debate 


\section{$V$. Deliberación}

Quien preside el jurado realiza una breve conclusión de los principales elementos que fueron discutidos durante la actividad, tratando de definir algunos consensos y plantear temas que aún contribuyen al debate nacional. Posteriormente comunica el veredicto final y argumenta brevemente su decisión.

\section{Anexo 4}

\section{Criterios de evaluación debate}

Considerando que las rondas persiguen objetivos diametralmente distintos, se propone definir criterios de evaluación diferentes que midan el estado de logro de cada estudiante. A continuación se define cada criterio de evaluación y su importancia relativa en cada etapa del debate.

\section{I) Criterios de evaluación de la primera ronda (40\%)}

1) Presentación personal e imagen

Cada estudiante deberá preocuparse de su apariencia física en términos de vestido e higiene personal. Se exige máxima formalidad en el vestuario para hombres y mujeres y preocupación por el orden de su barbilla (hombres) y cabello. Este criterio tiene una ponderación de un $10 \%$.

2) Lenguaje verbal

Se debe evitar el lenguaje coloquial y utilizar, en lo posible, la terminología asociada a la asignatura en evaluación. Se valora el uso de sinónimos y antónimos, así como la ausencia de muletillas; en general, se espera un buen uso del idioma. Este criterio tiene una ponderación de un $10 \%$.

3) Contenido

Este es el aspecto más importante de la evaluación de la primera ronda. Mide el nivel de conocimiento de sus participantes con respecto al tema, lo que se puede evidenciar en la cantidad de citas realizadas, en la documentación presentada, así como en la capacidad de vincular los contenidos vistos durante las asignaturas al aporte de soluciones a una problemática relevante. Este criterio tiene una ponderación de un 20\%.

\section{II) Criterios de evaluación de la segunda ronda (40\%)}

\section{1) Lenguaje no verbal}

Hace referencia al manejo de gestos y otros elementos del lenguaje corporal que refuerzan cada argumento en el momento de la exposición. En términos generales, Se evalúa, además, la capacidad para usar el espacio sin llegar a sobreactuaciones. Este criterio tiene una ponderación de un $10 \%$.

2) Coordinación y organización

Este aspecto mide el estado de logro de cada equipo en relación con su capacidad para trabajar colaborativamente. Se evalúan los implementos utilizados para organizarse así como también la coordinación en el momento de su presentación. Este criterio tiene una ponderación de un $10 \%$.

3) Debate de ideas

Este criterio persigue evaluar la capacidad estudiantil para exponer su desacuerdo en relación con argumentos del equipo contrario, y utilizar estos en defensa de su postura. Este criterio tiene una ponderación de un $20 \%$. 


\section{III) Criterios de evaluación ronda final (20\%)}

1) Síntesis y orden de ideas

Se espera que representantes de cada equipo logren exponer de forma ordenada los principales argumentos utilizados en defensa de su posición. Este criterio tiene una ponderación de un $10 \%$.

2) Debate de ideas

Este criterio persigue evaluar la capacidad que tiene el alumnado para exponer su desacuerdo en relación con argumentos del equipo contrario, y utilizar estos en defensa de su postura. Este criterio tiene una ponderación de un $10 \%$.

\section{Anexo 5}

\section{Pauta de evaluación}

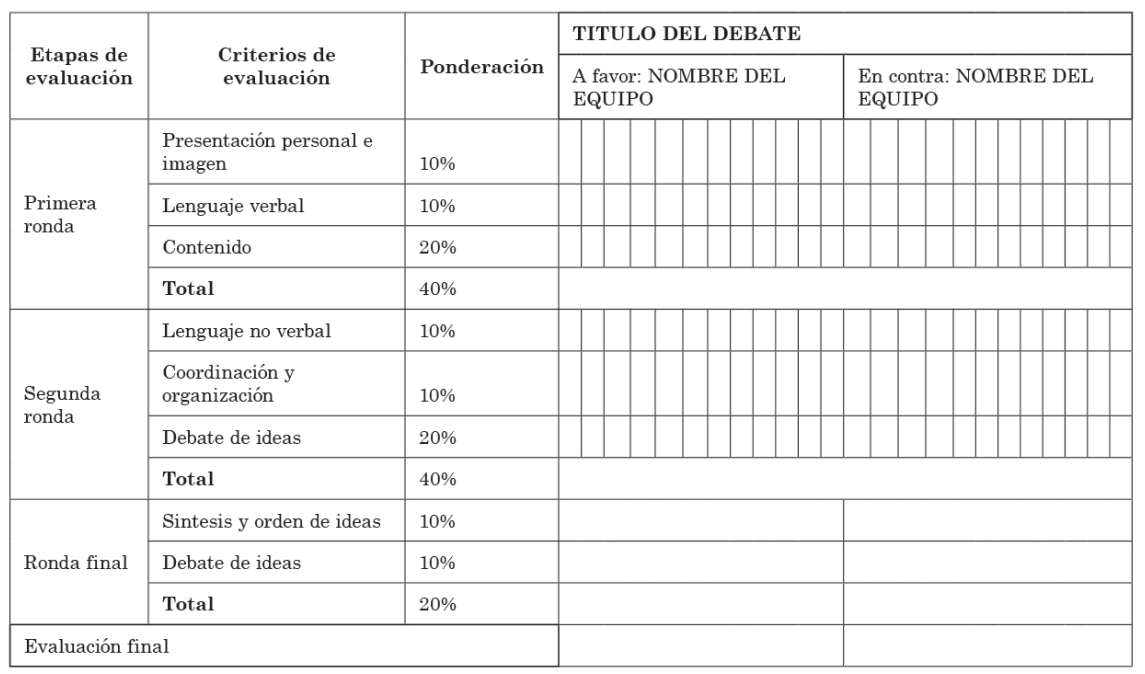

ANEXO 5

\section{Escala de apreciación}

\begin{tabular}{|l|l|l|}
\hline Tipo de logro & & Nota \\
\hline No logrado & 0 & 0 \\
\hline Bajo & 1 & 25 \\
\hline Promedio & 2 & 50 \\
\hline Bueno & 3 & 75 \\
\hline Excelente & 4 & 100 \\
\hline
\end{tabular}

ANEXO 5B 
Revista EdUCACión, 2018, 42(2), ISSN: 0379-7082 / 2215-2644

\section{Notas}

[1] Este trabajo es fruto del proyecto FDD2009-10 financiado por la Dirección de Docencia de la Universidad del Bío-Bío a través del Fondo de Desarrollo de la Docencia.

[4] El alumnado manifestó no conocerse entre sí como consecuencia de que provenían de generaciones distintas.

\section{BY-NC-ND}

